\title{
Capacitação profissional na Estratégia Saúde da Família: percepção dos enfermeiros
}

\author{
Professional training in the Family Health Strategy: perception of nurses \\ Formación profesional en la Estrategia de Salude de la Familia: percepción de las \\ enfermeras
}

Lázaro Clarindo Celestino ${ }^{1 *}$, Laura Adrian Leal ${ }^{1}$, Beatriz Regina da Silva ${ }^{1}$, Sônia Hutul Silva ${ }^{2}$, Beatriz Maria dos Santos Santiago Ribeiro ${ }^{1}$, Rita de Cássia de Marchi Barcelos Dalry ${ }^{1}$, Silvia Helena Henriques ${ }^{1}$.

\section{RESUMO}

Objetivo: Analisar a percepção de enfermeiros relacionada à capacitação profissional no âmbito da Estratégia Saúde da Família e identificar possíveis estratégias de capacitação oferecidas pelos gestores municipais. Método: Estudo exploratório de abordagem qualitativa. Participaram 18 enfermeiros das equipes da Estratégia da Saúde da Família de um município do interior de Minas Gerais. A coleta de dados ocorreu em julho de 2018 por meio de entrevistas semiestruturadas e posteriormente foi realizada análise temática indutiva. Resultados: Identificou-se deficiência de capacitação profissional e dificuldade dos enfermeiros em realizarem suas atividades no cotidiano laboral, o que pode acarretar risco psicossocial no ambiente de trabalho dos enfermeiros pesquisados. Além disso, não foram identificadas estratégias por parte dos gestores para eliminar e/ou amenizar os efeitos decorrentes da insatisfação dos enfermeiros quanto a capacitação insuficiente. Conclusão: Este estudo pode promover a reflexão e compreensão por parte dos enfermeiros e gestores, acerca da importância de se proporcionar capacitação profissional de qualidade para os profissionais atuantes na Estratégia Saúde da Família. Em suma, trabalhadores bem treinados e/ou capacitados são fundamentais para garantir o melhor atendimento aos usuários destes serviços e para gozarem de melhores condições de saúde física e mental.

Palavras-chave: Capacitação profissional, Saúde da família, Enfermeiros.

\section{ABSTRACT}

Objective: To analyze the perception of nurses related to professional training within the scope of the Family Health Strategy and to identify possible training strategies offered by municipal managers. Method: Exploratory study with a qualitative approach. Eighteen nurses from the Family Health Strategy teams from a city in the interior of Minas Gerais participated. Data collection took place in July 2018 through semi-structured interviews and subsequently an inductive thematic analysis was carried out. Results: We identified a lack of professional training and difficulties for nurses to carry out their activities in their daily work, which can cause psychosocial risk in the work environment of the nurses surveyed. In addition, no strategies were identified by managers to eliminate and / or mitigate the effects of nurses' dissatisfaction with. Insufficient training. Conclusion: This study can promote reflection and understanding on the part of nurses and managers, about the importance of providing quality professional training for professionals working in the Family Health Strategy. In short, well-trained, and / or qualified workers are fundamental to guarantee the best service to the users of these services and to enjoy better physical and mental health conditions.

Key words: Professional training, Family health, Nurses.

\section{RESUMEN}

Objetivo: Analizar la percepción de las enfermeras relacionadas con la capacitación profesional dentro del alcance de la Estrategia de Salud de la Familia e identificar posibles estrategias de capacitación ofrecidas por los administradores municipales. Método: estudio exploratorio con enfoque cualitativo. Participaron 18 enfermeras de los equipos de Estrategia de Salud Familiar de un municipio del interior de Minas Gerais. La recolección de datos tuvo lugar en julio de 2018 a través de entrevistas semiestructuradas y, posteriormente, se realizó un análisis temático inductivo. Resultados: Identificamos una falta de capacitación profesional y dificultades para que las enfermeras realicen sus actividades en su trabajo diario, lo que puede causar un

1 Universidade de São Paulo (USP), Ribeirão Preto - SP. *E-mail: lazaroenf@usp.br

¿Universidade Estadual de Londrina (UEL), Londrina - PR. 
riesgo psicosocial en el entorno laboral de las enfermeras encuestadas. Además, los gerentes no identificaron estrategias para eliminar y / o mitigar los efectos de la insatisfacción de las enfermeras con una capacitación insuficiente. Conclusión: Este estudio puede promover la reflexión y la comprensión por parte de enfermeras y gerentes, sobre la importancia de proporcionar capacitación profesional de calidad para los profesionales que trabajan en la Estrategia de Salud de la Familia. En resumen, los trabajadores bien capacitados y / o calificados son esenciales para garantizar la mejor atención para los usuarios de estos servicios y disfrutar de mejores condiciones de salud física y mental.

Palabras Clave: Formación profesional, Salud familiar, Enfermeras.

\section{INTRODUÇÃO}

A Estratégia Saúde da Família (ESF) foi criada com o objetivo de reorganizar o modelo de atenção à saúde no Brasil. Passou então, a ser considerada como porta prioritária de acesso do usuário ao sistema, tendo como norte os princípios organizacionais e doutrinários do Sistema Único de Saúde (SUS). O Ministério da Saúde determina uma equipe mínima multiprofissional de trabalho, composta por médicos, enfermeiros, auxiliares e/ou técnico de enfermagem, agentes comunitários de saúde, odontólogo e auxiliar e/ou técnico em saúde bucal (BRASIL, 2017; MIRANDA L, et al., 2019).

No contexto da ESF, os enfermeiros são os profissionais responsáveis por liderar a equipe, a eles são atribuídas diversas funções, como: realizar assistência integral aos indivíduos e família, realizar consultas de enfermagem; solicitar exames complementares e prescrever medicações de acordo com protocolo institucional; planejar, gerenciar e coordenar as atividades desenvolvidas pelos agentes comunitários de saúde (ACS), coordenar e realizar atividades de educação permanente, dentre outras (BRASIL, 2017). Assim, pode-se afirmar que constituem peça fundamental que integra a equipe multiprofissional.

Ademais, o excesso de atribuições e/ou atividades impostas pelas diretrizes da ESF e a falta de investimentos em capacitação profissional neste setor, contribuem para que os enfermeiros se sintam despreparados, gerando situações desgastantes no cotidiano de seu processo de trabalho, deixando-os, expostos a riscos ocupacionais, podendo então, acarretar prejuízo físico e mental a este trabalhador (MOREIRA KS, et al., 2017).

Para atuar no complexo cenário da ESF, é necessário a presença de profissionais atualizados e preparados, com competências específicas a este setor, para que estes possam atender às demandas dos usuários desse serviço (LOPES OCA, et al., 2020). Assim, com relação ao trabalho desenvolvido nesta realidade se faz necessário maior comprometimento dos gestores em garantir capacitações, especialmente aos enfermeiros, de forma a assegurar a qualidade da assistência prestada e melhores condições de trabalho.

Nessa linha de raciocínio, a compreensão da capacitação profissional dos enfermeiros no âmbito da ESF pode ser forte subsídio social e profissional, contribuindo para o planejamento de estratégias que visem diminuir os agravos à saúde do trabalhador, promovendo seu bem-estar físico e psicológico. Assim, este estudo apresenta os seguintes questionamentos: Qual a percepção dos enfermeiros quanto à capacitação para sua atuação na ESF? Os gestores municipais têm facilitado o oferecimento de treinamentos e/ou capacitações aos enfermeiros da ESF?

Para responder a estes questionamentos este estudo objetivou analisar a percepção de enfermeiros relacionada a capacitação profissional no âmbito da Estratégia Saúde da Família e identificar possíveis estratégias de capacitação oferecidas pelos gestores municipais.

\section{MÉTODOS}

Trata-se de um estudo exploratório de natureza qualitativa, realizado em 18 unidades da ESF de uma cidade do interior de Minas Gerais. Estas unidades estão distribuídas em pontos estratégicos da cidade e são responsáveis por território sanitário definido, sendo cada equipe responsável por aproximadamente 3.000 pessoas.

Assim, participaram da pesquisa os 18 enfermeiros atuantes nestas unidades da ESF do município em estudo. O critério de inclusão utilizado foi ser enfermeiro que exercia suas atividades na ESF há mais de três meses do início da coleta de dados. 
Para a coleta de dados realizou-se entrevistas semiestruturadas, gravadas em áudio e que posteriormente foram transcritas. Utilizou-se roteiro validado semanticamente com seis enfermeiros especialistas da área, o qual contém duas partes: a primeira diz respeito a questões referentes a dados de caracterização dos participantes como idade, sexo, ano de conclusão da graduação e tempo de atuação na instituição. Já, a segunda parte abordou questões abertas referentes à investigação da capacitação profissional na perspectiva do risco psicossocial.

Para a análise dos dados das entrevistas foi utilizada a análise de conteúdo indutiva para interpretação dos depoimentos dos participantes desse estudo. Optou-se pela utilização da análise temática, uma vez que é um método que identifica, analisa e relata os padrões (temas) dentro dos dados; organiza e descreve seu conjunto de dados em detalhes.

Nesse sentido, foram percorridas as seguintes fases: familiarização com os dados, geração de código, busca por temas, revisão de temas, definição e nomeação de temas e por último, a produção do relatório da análise (BRAUN V e CLARKE V, 2006). Por fim, encerrou-se a pesquisa mediante a saturação de informações, ou seja, quando ocorreu a convergência das falas, representada pela repetição sucessiva do conteúdo dos depoimentos.

O projeto foi aprovado pelo Comitê de Ética em Pesquisa da Escola de Enfermagem de Ribeirão Preto da Universidade de São Paulo, sob o protocolo OFíCIO nº 090/2018 e CAAE 83419418.2.0000.5393. Todos os participantes assinaram o Termo de Consentimento Livre e Esclarecido e para preservar o anonimato após as falas foi utilizado a letra $\mathrm{E}$ seguida pelo número arábico, sugestivo da ordem de realização das entrevistas (exemplo: E1).

\section{RESULTADOS}

Em relação à caracterização da população pesquisada, houve predominância do sexo feminino, representando 16 dos participantes (89\%), dado este já pressuposto devido à característica histórica da profissão. Com relação à idade, 12 participantes (67\%) se encontravam na faixa etária entre 36 e 40 anos. Quanto ao tempo de formação universitária, nove enfermeiros $(50 \%)$ possuíam formação profissional entre 11 e 15 anos e 12 profissionais (67\%) estavam atuando na ESF há mais de 11 anos. Outras variáveis podem ser observadas na Tabela 1.

Tabela 1 - Caracterização dos enfermeiros, segundo sexo, idade, tempo de formação na enfermagem e tempo de atuação na ESF, n=18. Ribeirão Preto, 2018.

\begin{tabular}{lcc}
\hline Caracterização dos Profissionais & $\begin{array}{c}\text { Enfermeiros } \\
\mathbf{N = 1 8}\end{array}$ & $\begin{array}{c}\text { Porcentagem } \\
\%\end{array}$ \\
\hline Sexo & & 89 \\
\hline Feminino & 16 & 11 \\
\hline Masculino & 02 & 5 \\
\hline Idade & & 5 \\
31 a 30 & 01 & 67 \\
36 a 40 & 01 & 11 \\
41 a 45 & 12 & 6 \\
46 a 50 & 02 & 6 \\
51 a 55 & 01 & 11 \\
\hline Tempo de formação na Enfermagem & 01 & 50 \\
06 a 10 anos & & 39 \\
11 a 15 anos & 02 & \\
16 a 20 anos & 09 & 17 \\
\hline Tempo de atuação na ESF & 07 & 67 \\
Menos de 1 ano & & 5 \\
11 a 15 anos & 03 & \\
16 a 20 anos & 02 & \\
\hline
\end{tabular}

Fonte: Celestino LC, et al., 2020. 
Em relação ao preparo e formação profissional, os resultados evidenciaram que 12 dos profissionais (67\%) apresentavam nível de pós-graduação, sendo que dentre esses 12, todos possuíam título de especialização lato sensu e $2(11 \%)$ ainda possuíam título de mestrado (stricto sensu). As áreas de formação dos enfermeiros na Especialização podem ser observadas na Tabela 2.

Tabela 2 - Distribuição dos enfermeiros, segundo o nível e a área de formação na pós-graduação em uma equipe da ESF, n=18. Ribeirão Preto, 2018.

\begin{tabular}{lcc}
\hline $\begin{array}{c}\text { Caracterização } \\
\text { da formação }\end{array}$ & $\begin{array}{c}\text { Enfermeiros } \\
\mathbf{N}=\mathbf{1 8}\end{array}$ & $\begin{array}{c}\text { Porcentagem } \\
\%\end{array}$ \\
\hline Nível de pós-graduação & & 56 \\
\hline $\begin{array}{l}\text { Lato Sensu } \\
\text { Especialização }\end{array}$ & 10 & 11 \\
$\begin{array}{l}\text { Stricto Sensu } \\
\text { Mestrado }\end{array}$ & 02 & 00 \\
Doutorado & 00 & $\%$ \\
\hline Especialização - Lato Sensu & $\mathbf{N = 1 2}$ & 17 \\
\hline Saúde Pública & 02 & 42 \\
Saúde da Família & 05 & 17 \\
Oncologia & 02 & 25 \\
Educação & 03 & 8 \\
Adm. Hospitalar e Gestão Pública & 01 & 8 \\
Obstetrícia/Estética & 01 & 17 \\
Urgência e Emergência & 02 & 8 \\
Gestão do SUS & 01 & 8 \\
Saúde do Trabalhador & 01 & 8 \\
Capacitação Pedagógica em & 01 & 8 \\
Enfermagem & & 17 \\
Administração Hospitalar & 01 & $\%$ \\
Saúde Mental & 02 & 100 \\
\hline Mestrado / Stricto Sensu & $\mathbf{N}=\mathbf{0 2}$ & \\
\hline Ciências da Saúde & 02 & \\
\hline Fon & &
\end{tabular}

Fonte: Celestino LC, et al., 2020.

Em relação aos discursos dos enfermeiros, a análise permitiu identificar ausência de investimento da instituição para a capacitação, formação e preparo desses trabalhadores para atuarem na ESF:

“[...] Não recebi nenhum treinamento... Nada específico ao programa de Saúde da Família ou de como desenvolver o trabalho do enfermeiro na Saúde da Família (E3)

"[...] Eu não tive nenhum preparo. Foi tudo assim, aprendendo no dia a dia mesmo. Fui lendo, estudando, porque não teve nenhum treinamento, nenhum curso, nem nada." (E18)

Ainda no universo da capacitação profissional, observou-se que muitos enfermeiros se utilizam de suas experiências de trabalhos anteriores para executarem suas atividades atuais, já que não receberam nenhum treinamento da instituição no início de suas atividades.

"[...] Eu já trabalhava na área hospitalar, trabalhei em vários hospitais, então eu trouxe de lá para cá um pouco da minha vivência. Assim deu pra agregar um pouco... Deu pra começar, aí estou aprendendo aos poucos como é o trabalho do enfermeiro na ESF." (E17)

"[...] Esta é minha segunda experiência de trabalho na Saúde da Família, atuei em outro município. Eu vim trabalhar aqui com base em minha experiência do outro município." (E16) 
Nesta direção, encontramos nesta pesquisa, um profissional que também menciona sobre a importância de a capacitação profissional acontecer antes de adentrarem o serviço, para que assim possam estar preparados a prestar um cuidado integral ao usuário em conformidade aos princípios doutrinários do SUS.

“[...] Quando eu entrei nesta unidade não tinha experiência alguma... Também não tive nenhum treinamento para exercer essa função. Então eu fico aqui pensando, os treinamentos deveriam acontecer imediatamente quando somos contratadas" (E11)

Assim, acredita-se que a capacitação profissional possa ser obtida por meio de especializações lato sensu e estágios supervisionados durante a graduação, bem como estágios como pre-trainee após graduação.

"[...] Quando eu entrei nesta unidade... Eu inclusive já tinha feito até a especialização em Saúde da Família” (E15)

"[...] Durante o último ano da faculdade, eu fiz estágio como pre-trainee na Santa Casa e depois fui pre-trainee nos PSF." (E8)

"[...] No último ano da faculdade eu fiz pre-trainee, porém foi no hospital" (E18)

Entretanto, alguns enfermeiros revelaram que a instituição oferece de forma esporádica alguns cursos de atualização de curta duração, na maioria das vezes, teóricos, preconizados pelo Ministério da Saúde, com os seguintes temas: Diabetes Mellitus, Saúde da Mulher, Saúde da Criança, Preventivo Papanicolau, Pré-natal, Hanseníase, Tuberculose, dentre outros.

"[...] Com o passar dos anos a gente treinou SIS pré-natal e SUS." (E 13)

"[...] A gente teve sobre diabetes, hanseníase, saúde materno infantil, saúde do trabalhador, da criança e etc." (E12)

"[...] Os cursos são direcionados a programas especificos, saúde da mulher, saúde da criança...” (E7)

"[...] Quando eu cheguei aqui entrei em pânico, pois foi meu primeiro emprego na área da saúde. Eu fiquei sem saber o que fazer, eu não sabia nem mesmo onde ficavam os materiais. Daí eu liguei pra minha chefe e falei, manda alguém pra me ajudar." (E5)

"[...] Recentemente passamos a ter alguns cursos teóricos, mas não considero como uma capacitação pois não tem a parte prática. Assim fica incompleto, pois a prática é fundamental né." (E8)

"[...] Eu tive alguns cursos teóricos aqui..., mas na faculdade eu tive o módulo de saúde da família, que deu um bom suporte para executar meu trabalho." (E10)

\section{DISCUSSÃO}

A caracterização dos participantes da pesquisa mostra que houve predominância do sexo feminino, representando 16 dos participantes (89\%), dado histórico da profissão. Pesquisa recente aponta que as mulheres representam $84,6 \%$ da força de trabalho na enfermagem brasileira, enquanto os homens somam apenas $15 \%$. Destaca-se ainda, que apesar desta disparidade de gênero, o número de homens na profissão, vem crescendo de forma considerável, desde a década de 1990 (COFEN e FIOCRUZ, 2016).

Em relação à capacitação profissional em nível de pós-graduação Stricto Sensu, dois enfermeiros possuíam mestrado em Ciências da Saúde. Deste modo, acredita-se na importância deste dado, haja visto que os Programas de Pós-Graduação stricto sensu constituem uma poderosa ferramenta de capacitação de profissionais da saúde e de várias outras áreas do conhecimento, buscando sempre respostas a determinado problema da sociedade como um todo. 
Destaca-se que 33\% dos profissionais não possuíam formação nível de pós-graduação, porém espera-se que a troca de experiências entre os profissionais no cotidiano de trabalho, contribua para a construção de conhecimentos entre si, tornando assim, um ambiente de trabalho saudável. Inicia-se um movimento, onde há a ruptura das práticas dos profissionais formados pelo modelo biomédico. Assim, este movimento de renovação e transformação das práticas propiciadas pelas especializações, proporcionam aos profissionais melhores condições de atuarem na perspectiva do cuidado integral, em consonância com os princípios doutrinários do SUS (ANDRADE RS, et al., 2016).

Destaca-se ainda que oito (67\%) dos enfermeiros pesquisados possuíam título de especialista nas áreas de Saúde da Família e Educação. A nível stricto sensu, a formação em Ciências da Saúde foi unânime, em contrapartida no lato sensu percebeu-se uma diversidade nas áreas de especializações. Este resultado representou um importante indicador de qualidade, pois demonstrou que os enfermeiros estão intensamente em busca de conhecimento, com o intuito de melhorar a prestação dos cuidados aos usuários, haja vista, a complexidade do trabalho nas ESF.

Estudo recente identificou a necessidade de capacitação profissional no nível de pós-graduação lato sensu e treinamentos no contexto da Atenção Primária a Sáude e Estratégia Saúde da Família. Entende-se que a carência em treinamentos destes profissionais, podem comprometer sobremaneira os princípios e atributos da Atenção Primária. Neste sentido, torna-se extremamente necessário, que gestores deste serviço, garantam um mínimo de programas de educação continuada e permanente aos enfermeiros deste setor (OLIVEIRA MM e PEDRAZA DF, 2019).

Para atuação na ESF, os enfermeiros necessitam de um arcabouço de competências específicas ao setor. Neste sentido a capacitação se faz primordial e pode ser definida como preparação ou habilitação do trabalhador para desempenhar determinada função. Assim, entendemos que a possibilidade de capacitação deve ser real no âmbito das instituições de Saúde, logo após a contratação dos servidores. Além disso, é fundamental que as instituições de saúde tenham seus objetivos bem definidos, trabalhadores preparados e seguros para executarem a função que Ihes foi confiada (ARAÚJO ST e PENAFORTE KL, 2016).

Em relação a ESF acredita-se também que o enfermeiro deva ser capaz de realizar diagnósticos, elaborar e executar planos de cuidados, investigar os determinantes e indicadores do processo saúde - doença da população. Assim, o preparo do profissional é essencial para a qualidade da assistência ao indivíduo e consequentemente para a melhora dos resultados organizacionais (ARAÚJO ST e PENAFORTE KL, 2016).

Neste sentido, destaca-se a necessidade destes profissionais em buscarem o desenvolvimento de conhecimentos, habilidades e atitudes, ou seja, competências profissionais, por meio das capacitações no âmbito da ESF. Destarte, a qualificação profissional se torna fundamental para o surgimento de competências específicas, que Ihes auxiliem em um atendimento integral, de forma a contribuir com a identificação do perfil dos enfermeiros atuantes na ESF (JUNIOR WPM, et al., 2017).

A importância de qualificar e capacitar profissionais da saúde e aperfeiçoar suas habilidades considerando o ambiente laboral fez com que o Ministério da Saúde, por meio da Portaria GM/MS no 1.996/2007, criasse a Política Nacional de Educação Permanente em Saúde (PNEPS), que objetiva o uso do trabalho e a realidade local como espaço de aprendizagem para melhor execução dos serviços (BRASIL, 2007).

Ressalta-se que a educação permanente, é um processo de aprendizagem que ocorre no cotidiano das pessoas e das organizações, considerando a subjetividade dos trabalhadores e seus conhecimentos como forma de transformação das práticas profissionais e da própria organização do trabalho (DIELH EE e PELLEGRINE MA, 2014). Quanto a isso, pesquisadores relataram a importância da educação e capacitação do profissional para atuação em seu ambiente de trabalho e revelaram falta de diálogo entre as instituições formadoras e os órgãos responsáveis pela prestação de serviço. Dessa forma, a educação no contexto do trabalho não se faz com planejamento das atividades advindas da realidade e do cotidiano desses serviços, o que diminui a efetividade e a qualidade no processo de trabalho (VIANA DMS, et al., 2015).

Os depoimentos dos enfermeiros desta pesquisa, corroboraram com estudo realizado recentemente, e que demonstrou a educação permanente como indispensável, sendo extremamente importante para atender demandas e processos já estabelecidos na ESF. Além da educação permanente, o estudo destacou ainda, outras competências indispensáveis ao enfermeiros da ESF, como: Liderança, ética, comunicação, trabalho 
em equipe, cuidado à saúde, recursos materiais e gestão de pessoas, tomada de decisão e estratégias individuais e organizacionais para desenvolvê-las (LOPES OCA, et at., 2020).

Contextualizando de modo geral, os discursos dos participantes, a necessidade de educação permanente se faz necessário, visto que auxiliam diretamente os enfermeiros nos processos de construção e fixação do próprio conhecimento diante do trabalho desempenhado, proporcionando a capacidade de identificar e resolver questões na ESF (SIQUEIRA-BATISTA R, et al., 2015).

Uma pesquisa de cunho qualitativo desenvolvida em um município do extremo Sul de Santa Catarina, destacou claramente as dificuldades da equipe frente à resolutividade dos casos e foi mencionada a falta de capacitações que reflete significativamente na rede de atenção qualificada e eficaz (CAETANO PS, et al., 2016).

Vale mencionar que uma pesquisa do tipo descritivo-exploratório, de natureza qualitativa realizada em ESF no município de Aparecida de Goiânia estudou 16 profissionais de nível superior, sendo oito médicos e oito enfermeiros e demonstrou dados parecidos com os mencionados nesse estudo, ou seja, referiram falta de experiência profissional. (MORI EC e NAGHETTINI AV, 2016).

Na percepção dos enfermeiros pesquisados, ter experiência pode ser um diferencial para atuação na ESF. Neste sentido, estudo de Santos MC, et al. (2017); reforça a importância de treinamentos, capacitação, para os profissionais que atuam no Sistema Único de Saúde, antes mesmo que estes adentrem os serviços de saúde. Com esse entendimento devem envolver-se todos os tipos de aprendizados a esses profissionais, desde cursos de especializações a educação permanente, visando melhorias tanto ao trabalhador quanto ao paciente.

A ESF envolve uma multiplicidade de programas de prevenção de agravos à saúde nos diferentes ciclos da vida. Organizar treinamentos para o atendimento dos usuários deve ser premissa dos gestores desses serviços. No tocante ao profissional enfermeiro, há um vasto universo de complexas atividades e reponsabilidades.

Estas atividades envolvem a promoção da saúde, prevenção de doenças e reabilitação; abrangem a assistência direta ao usuário, cadastrados ou não em unidades de saúde, perpassa por todos os ciclos de vida, sem distinção de cor, raça ou credo. Dentre as atividades mais realizadas pelo profissional enfermeiro, destacam-se a consulta de enfermagem, visita domiciliar, liberação e dispensação de medicamentos, orientações de todas as situações, acolhimento, agendamento de consulta com outros profissionais, dentre outras (PAULA M, et al., 2014).

Destarte, existem ainda, as atividades de cunho administrativas ou gerenciais desta categoria profissional, tais como, alimentação de sistemas de informação, reuniões de equipe, reuniões para planejar e direcionar diversos tipos de campanhas, emissão de relatórios, busca ativa de usuários em sistemas de informação, discussões sobre casos clínicos da área, atendimento de reclamações, contato com usuários via telefone, solicitação e controle de materiais, insumos e equipamentos, dentre outros (BRASIL, 2012).

Neste cenário, os enfermeiros se despontam como os principais atores, sendo fundamentais para a prestação de um cuidado de excelência. Entretanto, na prática observa-se extrema desvalorização destes profissionais, especialmente no âmbito da Atenção Primária à Saúde, enfrentando condições de trabalho inadequadas, dentre estas, a ausência e/ou capacitação profissional insuficiente (CELESTINO LC, et al., 2019).

Neste contexto, acreditamos ser fundamental que as instituições de saúde, representadas por seus respectivos gestores, assumam sua responsabilidade e invistam em treinamentos e capacitações de seus profissionais, e, além disso, que este preparo seja oferecido antes do profissional se inserir no trabalho e não depois que o mesmo já esteja por anos atuando na função. Dessa forma, os centros formadores têm o papel de desenvolver estratégias de ensino que reforcem as funções dos futuros profissionais para que possam atuar com efetividade nos diversos serviços de Saúde da complexa realidade sanitária.

Essa pesquisa apresenta a limitação de ter sido realizada com um número pequeno de profissionais em uma única região do Brasil. Assim ressalta-se a importância da realização de outros estudos de forma a ampliar a quantidade e a abrangência para outros locais, com intuito de verificar se há divergências na 
percepção da capacitação profissional, de forma a ampliar o conhecimento sobre como ocorrem às atividades educacionais, treinamentos ou preparos de profissionais que atuam em ESF.

\section{CONSIDERAÇÕES FINAIS}

Apesar de os enfermeiros receberem treinamentos teóricos sobre determinados assuntos, relacionados ao cotidiano da ESF, os mesmos relataram deficiência das capacitações, por estas não conterem a parte prática. Em suma, especialistas da área, tem destacado a necessidade de capacitações de qualidade, de forma a abranger teoria e prática. A capacitação profissional insuficiente ou deficiente dos enfermeiros representa um grave problema à saúde dos assistidos nas ESFs, sendo desde o acompanhamento na saúde do recém-nascido até à saúde do idoso. Para dar conta desta demanda, é necessário que haja enfermeiros com habilidades técnicas e teóricas, capazes de entenderem e atenderem a complexa realidade sanitária brasileira além das diversas demandas da população.

\section{REFERÊNCIAS}

1. ANDRADE RS, et al. Processo de trabalho em unidade de saúde da família e a educação permanente Trabalho, Educação e Saúde, 2016; 14(2): 505-521.

2. ARAUJO ST, PENAFORTE KL. Riscos psicossociais relacionados ao trabalho: percepção dos profissionais de enfermagem. Revista de enfermagem UFPE on line, 2016; 10(11): 3831-9.

3. BRASIL. Ministério da Saúde. Política Nacional de Atenção Básica (PNAB). Brasília, DF: Departamento de Atenção Básica, 2012.

4. BRASIL. Ministério da Saúde. Política Nacional de Atenção Básica (PNAB). Brasília, DF: Departamento de Atenção Básica, 2017.

5. BRASIL. Ministério da Saúde. Portaria GM/MS n. 1996, de 20 de agosto de 2007. Dispõe sobre as diretrizes para a implementação da Política Nacional de Educação Permanente em Saúde e dá outras providências. Brasília, DF; 2007.

6. BRAUN V, CLARKE V. Using thematic analysis in psychology. Qualitative Research in Psychology, London, 2006; 3(2): 77-101.

7. CAETANO OS, et al. Conduta do enfermeiro frente aos conflitos éticos e bioéticos em área vulnerável na ESF. Saúde e Pesquisa, 2016, 9.2: 349-360.

8. CELESTINO LC,. et al. Working conditions of nurses in the Family Health Strategy. International Archives of Medicine, [S.I.], 2019; 12.

9. CONSELHO FEDERAL DE ENFERMAGEM E FIOCRUZ. PESQUISA INÉDITA TRAÇA O PERFIL DA ENFERMAGEM 2016. Disponível em: http://www.cofen.gov.br/pesquisa-inedita-traca-perfil-da-enfermagem_3. Acesso em: 8 mar. 2020.

10. DIEHL EE, PELLEGRINI MA. Saúde e povos indígenas no Brasil: o desafio da formação e educação permanente de trabalhadores para atuação em contextos interculturais. Cad Saúde Pública [Internet]. 2014, 30(4): 867-874.

11. JUNIOR WPM, et al. Competência profissional do enfermeiro para atuar na estratégia saúde da família: uma revisão integrativa. REAS, Revista eletrônica acervo saúde, 2017; Sup.9: S688-S694.

12. LOPES OCA, et al. Competências dos enfermeiros na saúde da família, Escola Anna Nery, 24(2) 2020.

13. MIRANDA L, SILVA LJ, SOUZA YF. Entre ausência de doença e cuidado possível: a saúde segundo usuárias da estratégia saúde da família. Trab. Educ. Saúde, Rio de Janeiro, 2019; 17(1):e0017404.

14. MOREIRA KS. et al. Avaliação da infraestrutura das unidades de saúde da família e equipamentos para ações na atenção básica. Cogitare Enfermagem, 2017; 22(2).

15. MORI EC, NAGHETTINI AV. Formação de médicos e enfermeiros da estratégia Saúde da Família no aspecto da saúde do trabalhador. Revista da Escola de Enfermagem da USP, 2016, 50.SPE: 25-31.

16. OLIVEIRA MM, PEDRAZA DF. Contexto de trabalho e satisfação profissional de enfermeiros que atuam na Estratégia Saúde da Família. Revista Saúde Debate. 2019.

17. PAULA MD, et al. Características do processo de trabalho do enfermeiro da estratégia de saúde da família. Revista Mineira de Enfermagem-REME, 2014; 18(2): 454-462.

18. SANTOS MC, et al. Processo de Trabalho do Núcleo de apoio à Saúde da Família (NASF): Importância da Qualificação Profissional. Sau. \& Transf. Soc., Florianópolis, 2017; 8(2): 060-069.

19. SIQUEIRA-BATISTA R, et al. (Bio)ética e Estratégia Saúde da Família: mapeando problemas. Saúde soc. São Paulo, 2015; 24(1): 113-128.

20. VIANA DM, et al. A educação permanente em saúde na perspectiva do enfermeiro na estratégia de saúde da família. R Enferm Cent O Min. 2015; 5(2): 1658-1668. 